CLINICAL STUDY

\title{
Individual multi-locus heterozygosity is associated with lower morning plasma cortisol concentrations
}

\author{
Lina Zgaga $^{1,2}$, Veronique Vitart ${ }^{3}$, Caroline Hayward $^{3}$, Darko Kastelan ${ }^{4}$, Ozren Polašek ${ }^{5}$, Miro Jakovljevic ${ }^{6}$, \\ Ivana Kolcic ${ }^{5}$, Zrinka Biloglav ${ }^{2}$, Alan F Wright ${ }^{3}$, Harry Campbell ${ }^{1, *}$, Brian R Walker ${ }^{7, *}$ and Igor Rudan ${ }^{1, *}$ \\ ${ }^{1}$ Centre for Population Health Sciences, University of Edinburgh, Teviot Place, Edinburgh EH8 9AG, UK, ${ }^{2}$ Department of Medical Statistics, Epidemiology \\ and Medical Informatics, University of Zagreb School of Medicine, 10000 Zagreb, Croatia, ${ }^{3}$ MRC Human Genetics Unit, Institute of Genetics and Molecular \\ Medicine, University of Edinburgh, Edinburgh EH4 2XU, UK, ${ }^{4}$ Department of Endocrinology, University of Zagreb School of Medicine, University Hospital \\ Center, 10000 Zagreb, Croatia, ${ }^{5}$ Department of Public Health, University of Split, 21000 Split, Croatia, ${ }^{6}$ Department of Psychiatry, University of Zagreb \\ School of Medicine, University Hospital Center, 10000 Zagreb, Croatia and ${ }^{7}$ Queen's Medical Research Institute, British Heart Foundation Centre for \\ Cardiovascular Science, University of Edinburgh, Edinburgh EH16 4TJ, UK
}

(Correspondence should be addressed to B Walker; Email: b.walker@ed.ac.uk; I Rudan; Email: irudan@hotmail.com)

*(H Campbell, B R Walker and I Rudan contributed equally)

\begin{abstract}
Objective: Stress is implicated as a risk factor for numerous illnesses in humans, putatively in part mediated by biological responses to stress, such as elevated cortisol concentrations. The theory of genetic homoeostasis suggests that individual heterozygosity facilitates compensation for environmental stresses. We hypothesized that heterozygosity ameliorates the biological response to a given level of perceived stress, reflected in lower plasma cortisol concentrations.

Design: We examined the role of heterozygosity in the association between perceived psychological stress and morning cortisol concentrations in 854 individuals from the isolated island of Vis, Croatia. Methods: Cortisol concentrations were measured in morning plasma samples. A total of 1184 autosomal microsatellite markers were genotyped and individual multi-locus heterozygosity (MLH) was calculated as the proportion of heterozygous markers. The General Health Questionnaire with 30 items (GHQ-30) was used to assess the degree of psychological distress.

Results: Mean MLH was $34.85 \pm 0.45 \%$ (range: 31.97-36.22\%). Psychological distress (GHQ Likert score $>31$ ) was more prevalent in women ( $37 \mathrm{vs} 18 \%$ in men, $P<0.0001$ ), in less educated people $(\beta=-0.35$ per year in school, $P<0.001)$ and in lower socio-economic classes $(\beta=-3.59$, $P<0.0001)$. Cortisol concentrations were positively associated with psychological distress $(\beta=2.20$, $P=0.01)$. In a regression model adjusted for age, BMI, education and GHQ-30 score, MLH was independently and inversely associated with morning plasma cortisol concentrations $(P=0.005)$. Conclusion: More heterozygous individuals, as measured by microsatellite markers, had lower morning plasma cortisol concentrations for a given level of perceived psychological stress. This may be important, as higher cortisol concentrations may increase the allostatic load and be associated with a higher risk of stress-related illness.
\end{abstract}

European Journal of Endocrinology 169 59-64

\section{Introduction}

Stress has been implicated as a risk factor for numerous illnesses and for mortality in humans $(1,2)$. However, large inter-individual differences in perception of stress, response to stress and stress-induced susceptibility to disease can alter the stress-to-illness pathway $(3,4)$. Relevant individual characteristics include both psychological factors, such as personality and previous experiences, and somatic differences including genetic make-up $(5,6)$.

The biological response to stress includes activation of the hypothalamic-pituitary-adrenal axis, which regulates the release of cortisol. Elevated cortisol concentrations contribute to adaptive changes in immune, cardiovascular and metabolic systems to maintain homoeostasis, a process called allostasis. However, sustained elevation of cortisol concentrations (as exaggerated in Cushing's syndrome) results in maladaptive changes, representing 'allostatic load'.

In 1954, Lerner (7) introduced the idea of genetic homoeostasis, hypothesizing that individual heterozygosity is important because it facilitates compensation for environmental stresses and enhances an individual's ability to maintain homoeostasis in changing environments. The advantages of increased locus and multi-locus heterozygosity (MLH) for disease resistance, longevity and fitness, especially when the organism is 
challenged, have been described for several species including humans $(8,9,10)$. However, the underlying mechanism for this 'heterozygosity-fitness correlation' is not clear, and the reported associations with infectious disease susceptibility and common complex disease risk factors are usually weak, perhaps due to the fact that most human populations are outbred with nearly uniform and high heterozygosity of individuals $(11,12,13,14)$.

A closely related and more frequently investigated research theme is inbreeding, which leads to decreased heterozygosity in offspring. The phrase inbreeding depression' reflects the negative effects of inbreeding and the general inferiority of the inbred offspring. Besides the well-characterized role in monogenic conditions, negative effects of inbreeding and decreased heterozygosity on fitness in humans have also been described for complex traits and diseases $(14,15,16)$. Because its detrimental consequences are well known among laymen and professionals, inbreeding is discouraged in most human societies and also in agricultural and farming practices. Interestingly, it has been suggested that rural-to-urban migration and progressive globalization in the past century have led to outbreeding and consequentially to an increase in individual heterozygosity, which may have a beneficial effect on a range of human traits and could in part account for improved health and longevity $(9,15,16,17,18)$.

We aimed to investigate the role of heterozygosity in the individual biological response to perceived psychological stress. We did so by examining the associations among psychological stress, morning cortisol concentrations and heterozygosity.

\section{Subjects and methods}

The Croatia-Vis study included 924 unselected individuals from the island of Vis, Croatia, aged 18-93 years, who were phenotyped for over 50 disease-related quantitative traits $(9,15,19)$. The study received appropriate ethical committee approval, and all participants gave written informed consent. A custom-made questionnaire was used to collect information on age, socio-economic status (self-reported, estimated in comparison with that of others as 'much worse', 'a bit worse', 'same', 'a bit better' or 'much better'), education (number of completed years of education), medication, chronic illness and ancestry. Blood samples were collected between 0830 and $0930 \mathrm{~h}$ in the research centre after an overnight fast and were used for genetic and biochemical analyses. Individual data on medication were screened to identify individuals on corticosteroid treatment or hormone replacement therapy.

Twenty-two participants were taking corticosteroid medication and were excluded from the study. Genetic data did not pass the quality-control procedures for further nine participants and cortisol concentration values were not available for a further 32 participants. Finally, we also excluded female participants on hormone replacement therapy $(n=7)$, yielding a final sample of 854 participants who were included in the analysis.

We used the General Health Questionnaire with 30 items (GHQ-30), a screening instrument for minor psychological disorders, to assess the degree of psychological distress. It was administered by a survey team with many years of experience. The Likert method was used for scoring GHQ-30, where answers "better than usual', 'like usual', 'worse than usual' and 'much worse than usual' are scored with 0,1, 2 and 3 points respectively. A GHQ-30 score above 31 is considered suggestive of psychological distress when applied to a general population $(20,21)$. We considered scores $>2$ S.D. above the mean in our study population (51 points) as indicative of severe psychological distress.

Plasma cortisol concentrations were measured using RIA (MP Biomedicals, Cambridge, UK) with an intraassay coefficient of variation (CV) of $5.1-7.0 \%$, an interassay CV of $6.0-7.9 \%$, and a lower limit of detection of $40 \mathrm{nmol} / \mathrm{l}$. In regression analyses, cortisol concentrations were corrected for relatedness, because our sample consisted of many related individuals, violating the independence of observation assumption that is required for the majority of statistical tests due to shared genetic factors. The GenABEL package for $\mathrm{R}$ allows an easy adjustment for relatedness by estimating pairwise kinship coefficients from the genome-wide data.

\section{DNA extraction and genotyping}

DNA was extracted from EDTA whole-blood specimens using the salting out procedure, stored at the MRC Human Genetics Unit (Edinburgh, UK), and genotyped at the Center for Medical Genetics, Marshfield Medical Research Foundation (USA). DNA samples were analyzed with 1184 autosomal markers comprising the microsatellite markers taken from the Marshfield Screening Set and indel markers (detailed at http:// research.marshfieldclinic.org/genetics).

Microsatellite markers were excluded from the analysis if genotyping failed in more than $10 \%$ of all the samples and if the locus was homozygous for all the participants. We also excluded individuals for whom fewer than 750 markers were successfully genotyped, because genotyping failure is more common for heterozygous markers (22).

\section{MLH and standardized MLH}

Data on all the microsatellite markers that passed quality control were used to calculate MLH for each individual $\left(\mathrm{MLH}_{\mathrm{i}}\right)$, that is, the proportion of heterozygous microsatellite loci among the ones that have been successfully genotyped: 


$$
\operatorname{MLH}(i)=\frac{N_{\text {heterozygous (i) }}}{N_{\text {genotyped (i) }}}
$$

Standardized MLH (SMLH) was calculated as MLH $_{i}$ divided by the average MLH of all other $n$ individuals, restricted to the set of loci successfully typed in the individual $i$.

$$
\operatorname{SMLH}(i)=\frac{\operatorname{MLH}(i)}{\frac{\sum_{j=1}^{n} \operatorname{MLH}(i)}{n}}
$$

SMLH ranged from 0.9 to 1.1 , with mean \pm s.D. $=$ $1.00 \pm 0.03$. Calculations were performed using the Rhh application for R (available at http://cran.r-project. org/web/packages/Rhh/index.html).

\section{Statistical analysis}

Statistical analysis was performed using R (http://www. r-project.org/). The $\chi^{2}$ test was used to analyse categorical variables. A multivariate regression model was constructed and morning cortisol concentration (as a continuous variable) corrected for relatedness was used as the outcome. Predictor variables used in the model were age, gender, BMI, GHQ-30 score and SMLH. To enable comparison between $\beta$-coefficients, variables were normalized to standard normal distribution and expressed as z-scores.

\section{Results}

A total of 854 participants (485 (57\%) females) were included, 499 from the Komiza village and 355 from the town of Vis. The mean age of the participants was $56.56 \pm 15.67$ years. The median duration of education was 11 years (interquartile range (IQR): 8-12 years). The duration of education was inversely associated with age $(P<0.0001)$ and was significantly longer in males $(P<0.0001)$.

The median GHQ-30 (Likert) score was 26 (IQR: 20-33). A total of 245 participants (179 (73\%) females) were categorized as suffering from psychological distress (GHQ-30 > 31) and 35 participants (25 (71\%) females) had severe psychological distress (GHQ-30 > 51). In a multivariate regression analysis, we found that the GHQ-30 score was significantly associated with gender (prevalence of psychological distress was higher in females $(36.9 \%)$ than in males $(17.9 \%), P<0.0001)$, age $(\beta$-coefficient $=0.13, P<0.001)$, duration of education (among those with 8 or less years of fulltime education, 137 individuals $(40 \%)$ were distressed, in contrast to $11(17 \%)$ among those with 16 or more years of schooling, $P<0.001)$ and socio-economic status (prevalence of psychological distress in five categories of worst-to-best socio-economic status: 70 , 43, 27, 18 and 17\%, $P<0.0001)$.
Mean morning plasma cortisol concentration was $619.62 \pm 227.05 \mathrm{nmol} / \mathrm{l}$. There was no statistically significant difference in cortisol concentrations between males $(617.43 \pm 199.86 \mathrm{nmol} / \mathrm{l})$ and females $(621.28 \pm 245.92 \mathrm{nmol} / \mathrm{l}, P=0.1)$, but age $(P=0.003)$ and BMI $(P<0.001)$ were inversely associated with morning cortisol concentrations.

Mean MLH (including markers that passed quality control and were not homozygous in all individuals) was $34.85 \pm 0.45 \%$, range $31.97-36.22 \%$ (Fig. 1). SMLH $($ mean $=1.00$ and s.D. $=0.03)$ was used in the regression analysis.

Descriptive data according to cortisol tertiles are given in Table 1. Standardized MLH was lowest in those with the highest cortisol concentrations in one-way ANOVA $(P=0.035)$ and when adjusted for age and sex $(P=0.015)$. An association between higher morning cortisol concentrations (adjusted for relatedness) and lower SMLH $(P=0.005)$ was also found in a regression model adjusted for age, GHQ-30 score and BMI (Table 2). The association remained largely unchanged after the addition of adjustment variables sex and socioeconomic status to the model.

\section{Discussion}

In this study, we have shown that heterozygosity, as measured by microsatellite markers, is significantly and inversely associated with morning plasma cortisol concentrations. The association remained statistically significant after adjustment for perceived psychological distress and other measured potential confounders. This raises the possibility that the variation in the concentration of plasma cortisol, including its response to

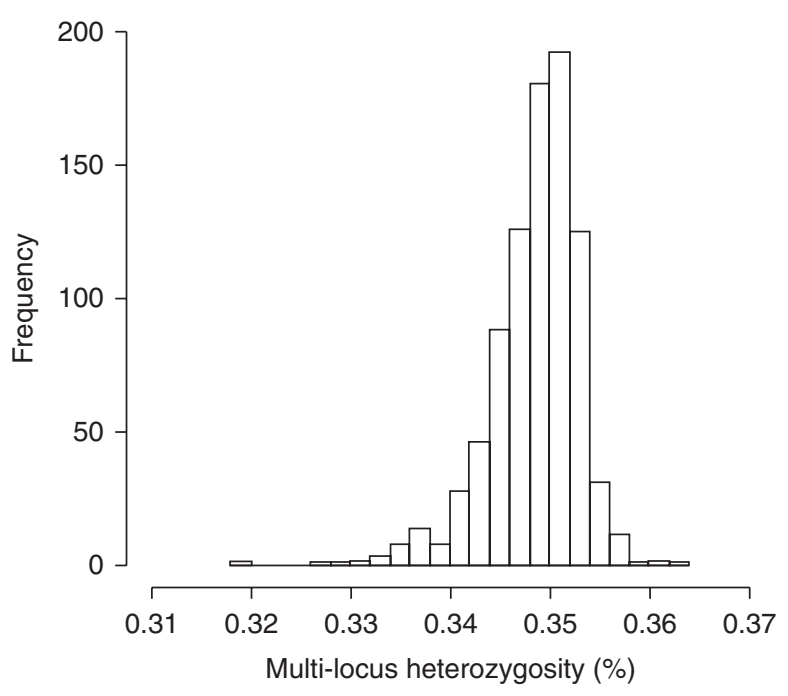

Figure 1 Histogram of multi-locus heterozygosity in the study population. 
Table 1 Characteristics of the participants by tertile of morning plasma cortisol concentrations. Data are number of participants (\%) or mean \pm s.D. values.

\begin{tabular}{|c|c|c|c|c|c|}
\hline & \multicolumn{5}{|c|}{ Cortisol } \\
\hline & All & $<508 \mathrm{nmol} / \mathrm{l}$ & $508-689 \mathrm{nmol} / \mathrm{l}$ & $\geq 690 \mathrm{nmol} / \mathrm{l}$ & $P^{a}$ (univariate) \\
\hline Cortisol (nmol/l) & $619.62 \pm 227.05$ & $400.36 \pm 82.49$ & $593.12 \pm 51.16$ & $865.94 \pm 188.19$ & \\
\hline$n$ (\% female) & 854 & $286(61.5)$ & $283(52.7)$ & $285(56.1)$ & $0.10^{\mathrm{b}}$ \\
\hline Age (years) & $56.6 \pm 15.7$ & $57.4 \pm 14.7$ & $58.2 \pm 16.0$ & $54.2 \pm 16.1$ & 0.003 \\
\hline GHQ-30 (Likert points) & $28.1 \pm 11.3$ & $27.7 \pm 10.7$ & $27.3 \pm 11.5$ & $29.2 \pm 11.5$ & 0.014 \\
\hline SMLH & $0.9995 \pm 0.0268$ & $0.9999 \pm 0.0288$ & $1.0009 \pm 0.0251$ & $0.9977 \pm 0.0263$ & 0.035 \\
\hline $\mathrm{BMI}$ & $27.3 \pm 4.2$ & $27.7 \pm 4.1$ & $27.6 \pm 4.2$ & $26.5 \pm 4.3$ & $<0.001$ \\
\hline Education duration (years) & $10.0 \pm 3.5$ & $10.0 \pm 3.5$ & $10.0 \pm 3.5$ & $9.9 \pm 3.6$ & \\
\hline$<9$ years $(n)$ & $3 \overline{48}$ & $1 \overline{11}$ & $11 \overline{1}$ & $1 \overline{2} 1$ & \\
\hline $9-12$ years $(n)$ & 364 & 124 & 124 & 116 & \\
\hline$>12$ years $(n)$ & 137 & 45 & 47 & 45 & $0.93^{b}$ \\
\hline Unknown & 5 & 1 & 1 & 3 & \\
\hline \multicolumn{6}{|l|}{ Socio-economic status } \\
\hline Much worse than that of others & 30 & 13 & 8 & 9 & \\
\hline A bit worse than that of others & 130 & 46 & 46 & 38 & \\
\hline Like that of others & 500 & 160 & 168 & 172 & \\
\hline A bit better than that of others & 171 & 54 & 57 & 60 & \\
\hline Much better than that of others & 19 & 10 & 3 & 6 & $0.53^{\mathrm{b}}$ \\
\hline Unknown & 4 & 3 & 1 & 0 & \\
\hline
\end{tabular}

GHQ-30, Generalized Health Questionnaire with 30 items; SMLH score, standardized multi-locus heterozygosity score.

${ }^{a} P$ value for the association from the one-way ANOVA (cortisol concentration analyzed as a continuous variable).

${ }^{\mathrm{b}} \chi^{2}$ test $P$ value for categorical variables (cortisol concentration analyzed by tertiles).

stressors and its contribution to allostatic load, is a mediator for the protective effect of heterozygosity on health.

The effect size of heterozygosity on cortisol concentrations was quite small (explaining $0.9 \%$ of the variance), but comparable to the effect of psychological distress found here $(1.5 \%)$ and in previous studies $(23,24,25,26)$. Moreover, for other biological variables, the percentage of variance explained by heterozygosity was typically in the range from 0.07 to $3.3 \%$ and $\sim 1 \%$ in studies with microsatellite markers (as has been used here) $(27,28,29)$. Also, given the marked circadian variation in plasma cortisol concentration, which peaks on waking from sleep, morning cortisol values are highly variable due to variations in the time of waking, stress response to venepuncture and precise time of sampling, so it is all the more remarkable that variation attributable to heterozygosity can be detected.

The characteristics of the cohort from the island of Vis may have contributed to the detection of these small effects in a modest number of participants. The GHQ-30 questionnaire has been shown to be a valid measure of acute psychological distress in the general population $(21,30)$, and we observed a similarly wide range of GHQ-30 scores among the study participants as in other studies. However, the GHQ score is not a 'general stress score' and other stressors may have an effect on an individual.

The distribution of morning plasma cortisol concentrations was also similar to that we observed in several other cohorts (unpublished data), and the association with GHQ-30 scores $(31,32,33)$ and other covariates including BMI (34) was as expected. However, most human populations today are outbred and individual heterozygosity is regularly found to be distributed in a narrow range of high values. Geographical isolation of the island of Vis and the limited immigration throughout its history are reflected in the pedigree structure of the study population $(15,35)$. A limited choice of sexual partners may have led to a higher degree of inbreeding in this isolated population, which is reflected in the varied levels of individual MLH, increasing the power of the regression analysis when heterozygosity is used as a covariate. Sharing of similar environment and lifestyle typical for island populations is also advantageous for the analysis, in that the participants are 'naturally matched' for a range of hidden influences that could confound the analysis.

Given the limited statistical power in this study, we were unable to explore whether heterozygosity contributes to the documented associations of plasma cortisol concentrations with the well-known risk factors for

Table 2 A multivariate regression analysis of morning plasma cortisol concentrations. $\beta$-Coefficients, $P$ values and percentage of variance explained are presented for the investigated predictor variables. Prior to analysis, covariates were normalized to standard normal distribution to enable the comparison of $\beta$-coefficients.

\begin{tabular}{lccc}
\hline & & & $\begin{array}{c}\text { Variance } \\
\text { explained (\%) }\end{array}$ \\
\hline Age & -0.56 & 0.001 & 1.1 \\
GHQ-30 & 0.39 & 0.009 & 1.5 \\
BMI & -0.5 & 0.001 & 1.3 \\
Standardized multi-locus & -0.41 & 0.005 & 0.9 \\
$\quad$ & & & \\
\hline
\end{tabular}


disease such as blood pressure and blood glucose (34). However, both in the current cohort (9) and in that of the Framingham Heart Study (11), heterozygosity was predictive of blood pressure, blood glucose, lipid profile and urate levels as well as left ventricular diameter and ventricular wall thickness.

The mechanism for the association of heterozygosity with cortisol concentrations is unknown. It is reasonable to infer a direction of causality from genetic constitution to biological variation rather than vice versa. Many mutations are recessive and exhibit effects only when homozygous. An individual with low heterozygosity is more likely to be homozygous for a number of deleterious recessive alleles. Recent genomewide association studies have shown that many common complex traits in humans are influenced by DNA sequence variants at multiple genetic loci (36). It therefore seems likely that homozygosity at numerous deleterious alleles (with individually small effects) may decrease the capacity to counterbalance stressors at an 'increased cost' in the form of increased cortisol concentrations and increased allostatic load.

On the other hand, there is the advantage of heterozygotes, or 'overdominance', which occurs when the heterozygote genotype has a higher relative fitness than either the homozygote mutant or the normal homozygote $(37,38)$. An example is that of sickle-cell anaemia in regions with prevalent malaria: homozygotes for mutant S-allele often die prematurely from sickle-cell disease and individuals with two normal alleles are susceptible to severe malaria, often with lethal outcomes, but heterozygous individuals are resistant to severe malaria and suffer none of the symptoms or mild symptoms of sickle-cell disease (39). By definition, less heterozygous individuals will have a smaller proportion of heterozygous loci, which will also include loci where the advantage of heterozygotes occurs. It seems likely that heterozygosity at some of the many loci likely to influence plasma cortisol concentrations is advantageous in this way. Identification of these loci, even for small effects, has the potential to flag pathways and mechanisms involved in the contribution of stress and cortisol concentrations to disease development.

Potential limitations of the study include the use of microsatellite markers to measure MLH as a proxy of true genome-wide heterozygosity. We followed previous recommendations for studying heterozygosity-fitness correlations in taking SMLH as a measure of heterozygosity and in investigating a fitness-related trait (40). However, tandem repeats are not thought to influence gene function directly (and are, therefore, not influenced by selection pressure directly), so we infer that heterozygosity of other genetic variants is mechanistically important in the protective effect, but we have not investigated those here.

Another limitation is that it is unknown how much time passed between waking and blood sampling for our participants, so not all would have been sampled at their peak cortisol concentrations (30-45 min after waking) (41) and cortisol concentrations might have been further elevated due to the stress related to the clinic visit and venepuncture. However, psychological distress has been shown to be associated with 'morning cortisol' concentrations measured in this way and not with just peak concentrations after waking $(31,32)$.

We conclude that in this cohort more heterozygous individuals had lower morning plasma cortisol concentrations for a given level of perceived psychological distress. This may be important as higher cortisol concentrations may increase the allostatic load and be associated with a higher risk of stress-related illness. In the future, alternative tools to SMLH, such as the analysis of 'runs of homozygosity', may be used to explore the generalizability of these findings in larger cohorts (42).

\section{Declaration of interest}

The authors declare that there is no conflict of interest that could be perceived as prejudicing the impartiality of the research reported.

\section{Funding}

This research did not receive any specific grant from any funding agency in the public, commercial or not-for-profit sector.

\section{Acknowledgements}

We thank Rosa Bisset for invaluable administrative support. We acknowledge the staff of several institutions in Croatia who supported the field work, including but not limited to The University of Zagreb Medical School and the Institute for Anthropological Research in Zagreb. We are grateful to Jill Harrison for technical assistance with cortisol measurements and to the British Heart Foundation and Chief Scientist Office of the Scottish Government for research funding.

\section{References}

1 Golden SH. A review of the evidence for a neuroendocrine link between stress, depression and diabetes mellitus. Current Diabetes Reviews 20073 252-259. (doi:10.2174/157339907782330021)

2 Matthews KA \& Gump BB. Chronic work stress and marital dissolution increase risk of posttrial mortality in men from the Multiple Risk Factor Intervention Trial. Archives of Internal Medicine 2002162 309-315. (doi:10.1001/archinte.162.3.309)

3 McEwen BS. Protective and damaging effects of stress mediators. New England Journal of Medicine 1998338 171-179. (doi:10. 1056/NEJM199801153380307)

4 McEwen BS \& Stellar E. Stress and the individual. Mechanisms leading to disease. Archives of Internal Medicine 1993153 2093-2101. (doi:10.1001/archinte.1993.00410180039004)

5 Caspi A, Sugden K, Moffitt TE, Taylor A, Craig IW, Harrington H, McClay J, Mill J, Martin J, Braithwaite A et al. Influence of life stress on depression: moderation by a polymorphism in the 5-HTT gene. Science 2003301 386-389. (doi:10.1126/science. 1083968)

6 Zhou Z, Zhu G, Hariri AR, Enoch MA, Scott D, Sinha R, Virkkunen M, Mash DC, Lipsky RH, Hu XZ et al. Genetic variation in human NPY expression affects stress response and emotion. Nature 2008452 997-1001. (doi:10.1038/nature06858) 
7 Lerner I. Genetic Homeostasis. Edinburgh: Oliver and Boyd, 1954.

8 Acevedo-Whitehouse K, Gulland F, Greig D \& Amos W. Inbreeding: disease susceptibility in California sea lions. Nature 200342235. (doi:10.1038/422035a)

9 Campbell H, Carothers AD, Rudan I, Hayward C, Biloglav Z, Barac L, Pericic M, Janicijevic B, Smolej-Narancic N, Polasek O et al. Effects of genome-wide heterozygosity on a range of biomedically relevant human quantitative traits. Human Molecular Genetics 200716 233-241. (doi:10.1093/hmg/ddl473)

10 Hellemann D, Larsson A, Madsen HO, Bonde J, Jarlov JO, Wiis J, Faber T, Wetterslev I \& Garred P. Heterozygosity of mannosebinding lectin (MBL2) genotypes predicts advantage (heterosis) in relation to fatal outcome in intensive care patients. Human Molecular Genetics 200716 3071-3080. (doi:10.1093/hmg/ ddm265)

11 Govindaraju DR, Larson MG, Yin X, Benjamin EJ, Rao MB \& Vasan RS. Association between SNP heterozygosity and quantitative traits in the Framingham Heart Study. Annals of Human Genetics 200973 465-473. (doi:10.1111/j.1469-1809.2009. 00514.x)

12 Lie HC, Simmons LW \& Rhodes G. Does genetic diversity predict health in humans? PLoS ONE 20094 e6391. (doi:10.1371/ journal.pone.0006391)

13 Lyons EJ, Amos W, Berkley JA, Mwangi I, Shafi M, Williams TN, Newton CR, Peshu N, Marsh K, Scott JA et al. Homozygosity and risk of childhood death due to invasive bacterial disease. BMC Medical Genetics 200910 55. (doi:10.1186/1471-2350-10-55)

14 Lyons EJ, Frodsham AJ, Zhang L, Hill AV \& Amos W. Consanguinity and susceptibility to infectious diseases in humans. Biology Letters 20095 574-576. (doi:10.1098/rsbl.2009.0133)

15 Rudan I, Biloglav Z, Vorko-Jovic A, Kujundzic-Tiljak M, Stevanovic R, Ropac D, Puntaric D, Cucevic B, Salzer B \& Campbell H. Effects of inbreeding, endogamy, genetic admixture, and outbreeding on human health: a (1001 Dalmatians) study. Croatian Medical Journal 200647 601-610.

16 Rudan I, Rudan D, Campbell H, Carothers A, Wright A, SmolejNarancic N, Janicijevic B, Jin L, Chakraborty R, Deka R et al. Inbreeding and risk of late onset complex disease. Journal of Medical Genetics 200340 925-932. (doi:10.1136/jmg.40.12.925)

17 Clark D. Urban World, Global City. Routledge, UK, 2004.

18 Rudan I \& Campbell H. Five reasons why inbreeding may have considerable effect on post-reproductive human health. Collegium Antropologicum 200428 943-950.

19 Vitart V, Rudan I, Hayward C, Gray NK, Floyd J, Palmer CN, Knott SA, Kolcic I, Polasek O, Graessler J et al. SLC2A9 is a newly identified urate transporter influencing serum urate concentration, urate excretion and gout. Nature Genetics 2008 40 437-442. (doi:10.1038/ng.106)

20 el-Rufaie OF \& Daradkeh TK. Validation of the Arabic versions of the thirty- and twelve-item General Health Questionnaires in primary care patients. British Journal of Psychiatry 1996169 662-664. (doi:10.1192/bjp.169.5.662)

21 Goldberg D. The Detection of Psychiatric Illness by Questionnaire. Oxford University Press, 1972.

22 Hirschhorn JN \& Daly MJ. Genome-wide association studies for common diseases and complex traits. Nature Reviews. Genetics 20056 95-108. (doi:10.1038/nrg1521)

23 Federenko IS, Nagamine M, Hellhammer DH, Wadhwa PD \& Wust S. The heritability of hypothalamus pituitary adrenal axis responses to psychosocial stress is context dependent. Journal of Clinical Endocrinology and Metabolism 200489 6244-6250. (doi:10.1210/jc.2004-0981)

24 Kajantie E \& Phillips DI. The effects of sex and hormonal status on the physiological response to acute psychosocial stress. Psychoneuroendocrinology 200631 151-178. (doi:10.1016/j.psyneuen. 2005.07.002)

25 Marques AH, Silverman MN \& Sternberg EM. Evaluation of stress systems by applying noninvasive methodologies: measurements of neuroimmune biomarkers in the sweat, heart rate variability and salivary cortisol. Neuroimmunomodulation 201017 205-208. (doi:10.1159/000258725)

26 Smyth J, Ockenfels MC, Porter L, Kirschbaum C, Hellhammer DH \& Stone AA. Stressors and mood measured on a momentary basis are associated with salivary cortisol secretion. Psychoneuroendocrinology 199823 353-370. (doi:10.1016/S0306-4530(98) 00008-0)

27 Chapman JR, Nakagawa S, Coltman DW, Slate J \& Sheldon BC. A quantitative review of heterozygosity-fitness correlations in animal populations. Molecular Ecology 200918 2746-2765. (doi:10.1111/j.1365-294X.2009.04247.x)

28 Coltman DW \& Slate J. Microsatellite measures of inbreeding: a meta-analysis. Evolution 200357 971-983.

29 David P. Heterozygosity-fitness correlations: new perspectives on old problems. Heredity $1998 \mathbf{8 0}$ (Pt 5) 531-537. (doi:10.1046/ j.1365-2540.1998.00393.x)

30 Goldberg D, Rickels K, Downing R \& Hesbacher P. A comparison of two psychiatric screening tests. British Journal of Psychiatry 1976 129 61-67. (doi:10.1192/bjp.129.1.61)

31 Clow A, Thorn L, Evans P \& Hucklebridge F. The awakening cortisol response: methodological issues and significance. Stress 20047 29-37. (doi:10.1080/10253890410001667205)

32 Ryff CD, Singer BH \& Dienberg Love G. Positive health: connecting well-being with biology. Philosophical Transactions of the Royal Society of London. Series B, Biological Sciences 2004359 1383-1394. (doi:10.1098/rstb.2004.1521)

33 Steptoe A, Wardle J \& Marmot M. Positive affect and health-related neuroendocrine, cardiovascular, and inflammatory processes. PNAS 2005102 6508-6512. (doi:10.1073/pnas.0409174102)

34 Walker BR. Glucocorticoids and cardiovascular disease. European Journal of Endocrinology 2007157 545-559. (doi:10.1530/EJE07-0455)

35 Rudan I, Campbell H \& Rudan P. Genetic epidemiological studies of eastern Adriatic Island isolates, Croatia: objective and strategies. Collegium Antropologicum 199923 531-546.

36 Lango Allen H, Estrada K, Lettre G, Berndt SI, Weedon MN, Rivadeneira F, Willer CJ, Jackson AU, Vedantam S, Raychaudhuri S et al. Hundreds of variants clustered in genomic loci and biological pathways affect human height. Nature $2010 \quad \mathbf{4 6 7} 832-838$. (doi:10.1038/nature09410)

37 Charlesworth D \& Charlesworth B. Inbreeding depression and its evolutionary consequences. Annual Review of Ecology and Systematics 198718 237-268. (doi:10.1146/annurev.es.18.110187. 001321)

38 Hansson B \& Westerberg L. On the correlation between heterozygosity and fitness in natural populations. Molecular Ecology 2002 11 2467-2474. (doi:10.1046/j.1365-294X.2002.01644.x)

39 Aidoo M, Terlouw DJ, Kolczak MS, McElroy PD, ter Kuile FO, Kariuki S, Nahlen BL, Lal AA \& Udhayakumar V. Protective effects of the sickle cell gene against malaria morbidity and mortality. Lancet 2002359 1311-1312. (doi:10.1016/S0140-6736(02) 08273-9)

40 Szulkin M, Bierne N \& David P. Heterozygosity-fitness correlations: a time for reappraisal. Evolution 201064 1202-1217.

41 Edwards S, Evans P, Hucklebridge F \& Clow A. Association between time of awakening and diurnal cortisol secretory activity. Psychoneuroendocrinology 200126 613-622. (doi:10.1016/ S0306-4530(01)00015-4)

42 McQuillan R, Eklund N, Pirastu N, Kuningas M, McEvoy BP, Esko T, Corre T, Davies G, Kaakinen M, Lyytikainen LP et al. Evidence of inbreeding depression on human height. PLoS Genetics 20128 e1002655. (doi:10.1371/journal.pgen.1002655)

Received 18 October 2012

Revised version received 17 February 2013

Accepted 1 May 2013 